\title{
Design of a nanostructured lipid carrier intended to improve the treatment of tuberculosis
}

\author{
This article was published in the following Dove Press journal: \\ Drug Design, Development and Therapy \\ 2 August 2016 \\ Number of times this article has been viewed
}

\author{
Marina Pinheirol,* \\ Ricardo Ribeiro 1 ,* \\ Alexandre Vieira ${ }^{1, *}$ \\ Fernanda Andrade ${ }^{2}$ \\ Salette Reis' \\ 'IUCIBIO, REQUIMTE, Chemistry \\ Department, Faculty of Pharmacy, \\ 'Laboratory of Pharmaceutical \\ Technology, Department of Drug \\ Sciences, Faculty of Pharmacy, \\ University of Porto, Porto, Portugal \\ *These authors contributed equally \\ to this work
}

\begin{abstract}
This work aimed to design, develop, and characterize a lipid nanocarrier system for the selective delivery of rifabutin (RFB) to alveolar macrophages. Lipid nanoparticles, specifically nanostructured lipid carriers (NLC), were synthetized by the high-shear homogenization and ultrasonication techniques. These nanoparticles were designed to exhibit both passive and active targeting strategies to be efficiently internalized by the alveolar macrophages, traffic to the acidified phagosomes and phagolysosomes, and release bactericidal concentrations of the antituberculosis drug intracellularly. NLC that could entrap RFB were prepared, characterized, and further functionalized with mannose. Particles' diameter, zeta potential, morphology, drug\% entrapping efficiency, and drug release kinetics were evaluated. The mannose coating process was confirmed by Fourier transform infrared. Further, the cytotoxicity of the formulations was evaluated by 3-(4,5-dimethylthiazol-2-yl)-2,5 diphenyltetrazolium bromide (MTT) assay in A549, Calu-3, and Raw 264.7 cells. The diameter of NLC formulations was found to be in the range of $175-213 \mathrm{~nm}$, and drug entrapping efficiency was found to be above $80 \%$. In addition, high storage stability for the formulations was expected since they maintained the initial characteristics for 6 months. Moreover, the drug release was $\mathrm{pH}$-sensitive, with a faster drug release at acidic $\mathrm{pH}$ than at neutral $\mathrm{pH}$. These results pose a strong argument that the developed nanocarrier can be explored as a promising carrier for safer and more efficient management of tuberculosis by exploiting the pulmonary route of administration.
\end{abstract}

Keywords: lipid nanoparticles, mannose, nanostructured lipid carriers, rifabutin, tuberculosis, drug delivery systems

\section{Introduction}

TB is an infectious disease caused by Mycobacterium tuberculosis (MTb). ${ }^{1}$ In 2015 , World Health Organization reported an estimated 9.6 million new cases of TB and 1.5 million deaths due to TB, making it the leading cause of death by infectious diseases in the world. ${ }^{2}$ The chemotherapy for the treatment of TB is extremely difficult due to the long treatment period and patient noncompliance, leading frequently to the emergence of multidrug resistant (MDR) strains. ${ }^{1}$ In this context, new and improved drug delivery strategies for existing drugs may play a crucial role in the TB management. Delivery systems for anti-TB drugs, including nanoparticles, may improve the therapeutic index of the antibiotics and, consequently, reduce the administration frequency and shorten the treatment duration. Moreover, an increase in patient compliance and efficacy of treatment, as well as a reduction in drug related toxicity is expected. ${ }^{3}$ Since their discovery in 1991, lipid nanoparticles have emerged as a potential alternative to other colloidal systems, including polymeric nanoparticles, since they combine their advantages and successfully overcome the main disadvantages attributed to them. ${ }^{4,5}$ Lipid 
nanoparticles show high drug encapsulation efficiency and high stability and do not require the use of organic solvents during production, thus being inexpensive to produce and easy to scale-up. ${ }^{4,6}$ In addition, lipid nanoparticles are made of endogenous lipids or lipids similar to those existing in the human body, and for this reason, they are considered biocompatible, biodegradable, and nontoxic. ${ }^{7}$ There are two main types of lipid nanoparticles, solid lipid nanoparticles (SLN) and nanostructures lipid carriers (NLC). ${ }^{5}$ SLNs only contains solid lipids, presenting perfect crystallinity. ${ }^{4}$ The perfect crystallinity frequently results in lower drug encapsulation efficiency, since there are few empty spaces into which the drug can be incorporated. ${ }^{5}$ It also results in drug's expulsion during long-term storage due to the changes in lipid packaging. NLC are a new generation of lipid nanoparticles that can overcome the drawbacks of SLN. ${ }^{5}$ NLC consist of solid and also liquid lipids, resulting in lower crystallinity, higher incidence of defects in the matrix, and a less dense lipid packaging. ${ }^{6}$ Thus, higher drug encapsulation efficiency and stability during long-term storage is achieved, in comparison with SLNs. ${ }^{5,6}$ Because of the aforementioned advantages of NLC over conventional SLN, NLC are starting to be exploited as nanocarriers in the TB field. ${ }^{8}$ In the TB treatment, RFB is commonly used, either as a second-line drug or as an alternative to the first-line anti-TB drug rifampicin in the case of resistance to rifampicin and in subjects coinfected with human immunodeficiency virus/MTb since it has fewer interactions with the antiretroviral drugs. ${ }^{9}$ RFB is a ryfamicin derivative with a large spectrum of antimicrobial activity, including activity against MTb. This drug binds to the $\beta$-subunit of RNA polymerase and inhibits RNA transcription and the protein synthesis of the bacteria. ${ }^{9}$

The present study aimed to design and develop NLC to selectively deliver RFB to the alveolar macrophages (AMs), where the etiological agent of TB is located, more specifically inside the acidic compartments of the phagosomes and phagolysosomes. ${ }^{3}$ Targeting the AMs with the designed nanocarrier was conceived to occur via two different strategies: passive and active targeting. NLC must possess the optimal mean aerodynamic diameter range for particle deposition in the lower airways (ie, between 1-3 $\mu \mathrm{M}$ and $1-100 \mathrm{~nm}$ ) and also to enable phagocytosis by the AMs $(>200 \mathrm{~nm}$ ), using passive targeting based on the natural propensity of macrophages to engulf particles. ${ }^{3}$ Moreover, the active targeting was also combined with the attachment of a ligand, namely mannose, which increases the selectivity of the nanocarrier to the AMs since receptors of mannose are highly expressed in the macrophages. ${ }^{10}$

\section{Materials and methods Materials}

NLC were prepared with Precirol ${ }^{\circledR}$ ATO 5 provided by Gattefossé (Nanterre, France), polysorbate 60 (supplied by Merck [Darmstadt, Germany]), and miglyol-812 from Acofarma (Madrid, Spain). RFB (more than 98\% pure), stearyl amine, Aerosil, D-(+)-Mannose (more than $99 \%$ pure), and (3-[4,5-dimethylthiazol-2-yl]-2,5 diphenyltetrazolium bromide) (MTT) were purchased from Sigma-Aldrich (St Louis, MO, USA). Double-deionized water used was obtained from a Millipore system with a conductivity less than $0.1 \mu \mathrm{S} \mathrm{cm}^{-1}$.

\section{Preparation of the formulations Preparation of the NLC}

The method chosen for the preparation of the NLC were the high-shear homogenization and ultrasonication techniques. The solid lipid (58\% w/w), the liquid lipid (25\% w/w), the surfactant $(16 \% \mathrm{w} / \mathrm{w})$, and the drug $(1 \% \mathrm{w} / \mathrm{w})$ were heated in a water bath up to $70^{\circ} \mathrm{C} .{ }^{11}$ The drug was added to a total amount of $7 \mathrm{mg}$. When the solid lipid was completely melted, $4.4 \mathrm{~mL}$ of heated ultrapure water $\left(T=70^{\circ} \mathrm{C}\right)$ was added to the mixture. The mixture was then passed through ultra-turrax T25 (IKA ${ }^{\circledR}$ Werke GmbH \& Co. KG, Staufen, Germany) at 3,500 rpm for 30 seconds, followed by sonication using a Sonics and Materials Vibra-Cell ${ }^{\mathrm{TM}} \mathrm{CV} 18$ (Sonics and Materials Inc., Newtown, CT, USA) at $70 \%$ power for 5 minutes, resulting in the formation of a nanoemulsion. The nanoemulsion was left to cool and stored at room temperature.

\section{Preparation of mannosylated nanostructures lipid carriers}

The coating of NLC with mannose was adapted from the method described by Jain et al. ${ }^{12}$ First, NLC were modified to exhibit amine groups at the surface through the addition of stearyl amine at a ratio of $2 \% \mathrm{w} / \mathrm{w}$. A mannose solution $(50 \mathrm{mM})$, prepared in acetate buffer $(\mathrm{pH}=4)$, was added to the NLC suspension and left under constant and gentle stirring for 48 hours. The acidic environment results in the ring opening of the mannose molecules, causing the aldehyde group to react with the free amine present at the surface of the stearyl amine functionalized NLC, resulting in the formation of the Schiff's base $(-\mathrm{N}=\mathrm{CH}-) .{ }^{13}$ Mannosylated nanoparticles were dialyzed (dialysis bag; Cellu.Sep ${ }^{\circledR} \mathrm{T} 3$ with a nomimolecular weight cut off of 12,000-14,000. [Membrane Filtration Products, Inc., Seguin, TX, USA]) against double-distilled water for 30 minutes to remove uncoated mannose. ${ }^{11}$ 


\section{Lyophilization}

Lyophilization was performed during the optimization process of the synthesis to improve the physical and microbiological stability of the formulations. The lyophilization protocol was adapted, with slight modifications, from the method described by Varshosaz et al. ${ }^{14} \mathrm{~A}$ VirTis freeze dryer (Advantage Plus EL-85; SP Scientific, Gardiner, NY, USA) was used for the lyophilization process. The samples were prepared with Aerosil $2 \%(\mathrm{w} / \mathrm{w})$ as cryoprotectant. The initial freezing was done at $-60^{\circ} \mathrm{C}$ for 720 minutes. The condensation process was performed at $-80^{\circ} \mathrm{C}$ under $150 \mathrm{mTorr}$ of pressure. The first drying was performed at $20^{\circ} \mathrm{C}$ for 1,200 minutes, under $150 \mathrm{mTorr}$ of pressure. A secondary drying was made at $25^{\circ} \mathrm{C}$, for 1,200 minutes, under $100 \mathrm{mTorr}$ of pressure.

\section{Characterization of the formulations}

\section{Encapsulation efficiency}

The drug encapsulation efficiency (EE) of nanoparticles was evaluated by measuring the absorption at the wavelength of $319 \mathrm{~nm}$ using ultraviolet (UV)/visible (VIS) spectrophotometry (Jasco, Easton, MD, USA). The lyophilized formulation samples with drug were diluted in double-deionized water (1:200) and centrifuged. The pellet was dissolved in $400 \mu \mathrm{L}$ of acetonitrile and vigorously vortexed for 30 minutes to completely extract the drug from the nanoparticles. Then, the resulting solution was transferred into Ultrafree ${ }^{\circledR}$ Centrifugal Amicon ${ }^{\circledR}$ Ultra-4 Centrifugal Filter Devices, with nominal molecular weight cutoff 10,000 kDa MWCO (Millipore, Billerica, MA, USA). Centrifugation was carried out using a Jouan BR4i multifunction centrifuge with a KeyWrite- ${ }^{\text {TM }}$ interface (Thermo Electron, Waltham, MA, USA) with a fixed $23^{\circ}$ angle rotor and $3,000 \times g$ spin for 8 minutes at $20^{\circ} \mathrm{C}$. The drug released from the nanoparticles was present in the supernatant, which was stored in the centrifuge tube until quantification by $\mathrm{UV} / \mathrm{V}$ is spectrophotometry. The EE of RFB was defined as the ratio between the quantity of RFB measured in the supernatant and initial quantity of drug added to the formulation. ${ }^{15}$

$$
\mathrm{EE}=\frac{\text { Total amount of RFB in nanoparticles }}{\text { Initial amount of RFB }} \times 100
$$

\section{Particles' diameter measurement}

Particles' diameter was measured by dynamic light scattering (DLS), using a BI-MAS DLS instrument (Brookhaven Instruments, Holtsville, NY, USA), operating at a scattering angle of $90^{\circ}$. Prior to the measurements, samples were diluted (1:100) in Milli-Q water and filtered with a syringe filter $(800 \mathrm{~nm})$. DLS data were analyzed at a temperature of $20^{\circ} \mathrm{C}$ with a dust cut-off set to 30 . The mean hydrodynamic diameter ( $Z$-average) and the polydispersity index (PDI) were determined as a measure of the width of the particles' diameter distributions. At each measurement, six runs of 2 minutes each were performed. The measurements were performed in triplicate.

\section{Zeta-potential measurement}

The $\zeta$-potential of the particles was determined by electrophoretic mobility using a BI-MAS DLS instrument (Brookhaven Instruments). Prior to the measurements, samples were diluted (1:100) in Milli-Q water and filtered with a syringe filter $(800 \mathrm{~nm})$. For each measurement, ten runs (each one with ten cycles) were performed at $20^{\circ} \mathrm{C}$. The measurements were performed in triplicate.

\section{FT-IR analysis}

Fourier transform infrared (FT-IR) analysis was performed on the lyophilized formulations to detect Schiff's base and confirm coating with mannose. FT-IR was performed with a PerkinElmer ${ }^{\circledR}$ spectrophotometer (Spectrum 400, Waltham, MA, USA) equipped with an attenuated total reflectance device and zinc selenite crystals. The samples were transferred to the attenuated total reflectance compartment, and the final result was obtained by combining the 32 scans. The spectra were recorded between 4,000 and $600 \mathrm{~cm}^{-1}$, with a resolution of $4 \mathrm{~cm}^{-1}$.

\section{In vitro drug release study}

The in vitro drug release studies were performed using a cellulose dialysis bag diffusion technique Dialysis bag (Cellu.Sep ${ }^{\circledR}$ T3 with a nomimolecular weight cut off of 12,000-14,000. [Membrane Filtration Products]) filled with $2 \mathrm{~mL}$ of the lyophilized samples, RFB-loaded NLC (NLC-RFB), and mannosylated RFB-loaded NLC (M-NLC-RFB).

An in vitro drug release study was performed using a direct dispersion method with appropriate buffers at $\mathrm{pH} 7.4$, 6.2 , and 5 to simulate the release of the drug following pulmonary administration until it reaches the acidic vesicles, phagosomes, and phagolysosomes, where the bacteria is located inside the AMs. To this end, the formulations were incubated at body temperature $\left(37^{\circ} \mathrm{C}\right)$ while being stirred in simulated physiological $\mathrm{pH}$ of the lung fluid (phosphate buffer, $\mathrm{pH}$ 7.4), the simulated physiological $\mathrm{pH}$ of the nasal fluid and phagosomes (phosphate buffer, $\mathrm{pH}$ 6.2), and also the simulated physiological $\mathrm{pH}$ of the phagolysosomes (phosphate buffer, $\mathrm{pH}$ 5.0). ${ }^{16}$ At regular intervals, $200 \mu \mathrm{L}$ of the solution was collected to quantify the drug release, and the same amount was substituted with the same volume 
of buffer to maintain sink conditions. The RFB release was determined using a plate reader at $319 \mathrm{~nm}$. The cumulative percentage of the released compound was determined using the average of the triplicates.

\section{Cytotoxicity}

The cytotoxicity of the lyophilized formulations was studied using MTT assay with three different cell lines, namely, Calu-3, A529 and Raw cells. Human airway epithelial cell line Calu-3 (ATCC ${ }^{\circledR}$ HTB-55 ${ }^{\mathrm{TM}}$, Rockville, MD, USA), human bronchial epithelial cell line A529 (ATCC ${ }^{\circledR}$ CCL-185 ${ }^{\mathrm{TM}}$ ), and murine macrophage RAW 264.7 cells (ATCC ${ }^{\circledR}$ TIB-71'TM) were cultured in Dulbecco's Modified Eagle Medium (DMEN) supplemented with 10\% (v/v) fetal bovine serum, $1 \%(\mathrm{v} / \mathrm{v})$ penicillin-streptomycin, and 1\% (v/v) Fungizone ${ }^{\circledR}$ antimycotic agent (Thermo Fisher Scientific Inc., Waltham, MA, USA). Cells were maintained in a humidified chamber at $37^{\circ} \mathrm{C}$ and $5 \% \mathrm{CO}_{2}$, and the culture medium was replaced every 2-3 days. Calu-3 and A549 cells were detached with a trypsin solution, while RAW 264.7 cells were detached using a scraper. The cell lines were harvested at $80 \%$ confluence.

Following exposure to the developed formulations, MTT assay was performed to assess cell viability. Briefly, Calu-3, A529, and RAW 264.7 cells were cultured in 96-well plates at a density of $2.5 \times 10^{4}$ cells $/ \mathrm{mL}$ for 24 hours before use. The following day, the culture medium was detached, and the lyophilized formulations were added at different concentrations (corresponding to $1-1,000 \mu \mathrm{g} \mathrm{mL}^{-1}$ ). Two controls were also tested, including cells treated with culture medium and cells treated with Triton ${ }^{\mathrm{TM}}$ X-100 (Sigma-Aldrich Co., St Louis, MO, USA) $2 \%(\mathrm{w} / \mathrm{v})$ in culture medium. After incubation for 24 hours, the culture medium was removed and replaced with $200 \mu \mathrm{L}$ of MTT diluted in fresh DMEM at $0.5 \mathrm{mg} / \mathrm{mL}$. The plate was incubated for 4 hours at $37^{\circ} \mathrm{C}$ without light. MTT solution was discarded, and formazan crystals were solubilized by addition of $200 \mu \mathrm{L}$ of dimethyl sulfoxide. The plate was shaken for 10 minutes at room temperature, and the absorbance (590 and $630 \mathrm{~nm}$ for background deduction) was measured using a Synergy ${ }^{\text {TM }}$ HT Multi-mode microplate reader (BioTek Instruments Inc., Winooski, VT, USA).

\section{Statistical analysis}

Statistical analysis was carried out using IBM $^{\circledR}$ SPSS $^{\circledR}$ statistics software version 21.0 (IBM Corporation, Armonk, NY, USA). The results are reported as the mean \pm standard deviation for a minimum of three independent experiments. Two-tailed Student's $t$-test and one-way analysis of variance (ANOVA) were performed to compare, respectively, two or multiple independent groups. If differences between the groups were found to be significantly different $(P<0.05)$, they were compared using the Tukey's post hoc test. The paired samples were analyzed through the paired-samples two-tailed Student's $t$-test. Differences were considered to be statistically significant at $P<0.05$.

\section{Results and discussion \\ Particles' diameter measurement}

The mean hydrodynamic particles' diameter for NLC and M-NLC (both unloaded and drug-loaded nanoparticles) are presented in Table 1. The mean particles' diameter of NLC and M-NLC unloaded nanoparticles was found to be $175 \pm 3$ and $198 \pm 2 \mathrm{~nm}$, respectively (Table 1). The incorporation of the RFB showed a size distribution with a mean diameter of $180 \pm 2$ for NLC-RFB and $213 \pm 2 \mathrm{~nm}$ for M-NLC-RFB, and no statistical differences were observed $(P>0.05)$, suggesting that RFB incorporation does not influence the nanoparticles diameter. On the other hand, the mannosylation process induced a significant increase in particles diameter $(P<0.05)$. Additionally, for all formulations, the PDI obtained was found to be $>0.1$ (Table 1), indicating that none of the formulations were monodisperse. Notwithstanding, the obtained diameter was within the breathable range, and therefore, the formulations have potential to reach the AMs. ${ }^{17}$ Moreover, it is expected that the functionalized nanoparticles with the mean diameter of around $200 \mathrm{~nm}$ and administered by pulmonary route will be suitable to reach and be internalized by the AMs. ${ }^{18}$

\section{Zeta-potential measurement}

As can be observed from the values given in Table 2, the unloaded NLC and the NLC loaded-RFB showed a pronounced negative average $\zeta$-potential of $-20.5 \pm 0.8$ and $-17.9 \pm 1.9 \mathrm{mV}$, respectively. Thus, the incorporation of RFB did not significantly change $(P>0.05)$ the surface charge

Table I Mean hydrodynamic diameter and PDI of NLC, M-NLC, NLC-RFB, and M-NLC-RFB formulations

\begin{tabular}{lll}
\hline Formulation & $\begin{array}{l}\text { Mean hydrodynamic } \\
\text { diameter }(\mathbf{n m})\end{array}$ & $\begin{array}{l}\text { Polydispersity } \\
\text { index (PDI) }\end{array}$ \\
\hline NLC & $175 \pm 3$ & $0.21 \pm 0.02$ \\
M-NLC & $198 \pm 2^{\mathrm{a}}$ & $0.12 \pm 0.0 \mathrm{I}^{\mathrm{a}}$ \\
NLC-RFB & $180 \pm 2$ & $0.12 \pm 0.02^{\mathrm{b}}$ \\
M-NLC-RFB & $213 \pm 2^{\mathrm{a}, \mathrm{c}, \mathrm{d}}$ & $0.12 \pm 0.02^{\mathrm{a}}$ \\
\hline
\end{tabular}

Notes: All values represent the mean \pm standard deviation $(n=3)$. ${ }^{\text {a }}$ tatistically

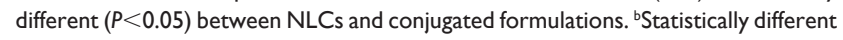
$(P<0.05)$ between unloaded and loaded NLCs. 'Statistically different $(P<0.05)$ from M-NLC, between conjugated and unloaded formulations. 'Statistically different $(P<0.05)$ between unloaded conjugated NLCs and RFB loaded conjugated NLCs. Statistically different $(P<0.05)$ between loaded NLCs nonconjugated and loaded NLCs conjugated.

Abbreviations: PDI, polydispersity index; NLC, nanostructured lipid carrier; M, mannose; RFB, rifabutin. 
Table 2 Characterization of the zeta-potential of the NLC, M-NLC, NLC-RFB, and M-NLC-RFB formulations

\begin{tabular}{ll}
\hline Formulation & Zeta-potential $(\mathbf{m V})$ \\
\hline NLC & $-20.5 \pm 0.8$ \\
M-NLC & $+48.7 \pm 2.1^{\mathrm{a}}$ \\
NLC-RFB & $-17.9 \pm 1.9$ \\
M-NLC-RFB & $+37.6 \pm 1.0^{\mathrm{a}-\mathrm{c}}$ \\
\hline
\end{tabular}

Notes: All values represent the mean \pm standard deviation $(n=3)$. aStatistically different $(P<0.05)$ between NLCs and conjugated formulations. 'SStatistically different $(P<0.05)$ between unloaded conjugated NLCs and RFB loaded conjugated NLCs. 'Statistically different $(P<0.05)$ between loaded NLCs nonconjugated and loaded NLCs conjugated.

Abbreviations: NLC, nanostructured lipid carrier; M, mannose; RFB, rifabutin.

of the lipid nanoparticles. On the other hand, statistically significant changes in $\zeta$-potential were observed with the mannosylation process $(P<0.05)$, since M-NLC and M-NLC-RFB exhibit a positive charge of $+48.7 \pm 2.1$ and $+37.6 \pm 1.0 \mathrm{mV}$, respectively. The values obtained strongly suggest successful mannosylation of the NLC because this process involves adding stearylamine to the formulations, so that nanoparticles have positively charged amine groups at the surface. The NLC with stearylamine exhibited a $\zeta$-potential charge of $+57.1 \pm 0.4$ (data not shown), confirming the successful addition of stearylamine, which provided the positively charged amine groups-tailored surfaces on NLC. The coating with mannose shields the positive charge, and hence, the $\zeta$-potential obtained was lower (Table 2), which suggests the successful binding of mannose to the amine groups. Moreover, the mannosylated NLC possess a high absolute $\zeta$-potential and are therefore considered physically stable due to the electrostatic repulsions between particles. ${ }^{19}$

\section{$\mathrm{EE}$}

Table 3 summarizes the EE obtained for RFB in the both plain and mannose-coated NLC. The RFB EE of NLC and M-NLC was found to be $83 \% \pm 6 \%$ and $90 \% \pm 4 \%$, respectively. Mannosylation did not significantly $(P>0.05)$ change the $\mathrm{EE}$, so the technique may be used without any reduction in the capacity of this nanocarrier to incorporate this drug. This highly satisfactory $\%$ of encapsulation confirms that NLC is a suitable nanocarrier for RFB incorporation. This

Table 3 Drug's encapsulation efficiency of the NLC-RFB and M-NLC-RFB formulations

\begin{tabular}{ll}
\hline Formulation & $\begin{array}{l}\text { Drug encapsulation } \\
\text { efficiency (\%) }\end{array}$ \\
\hline NLC-RFB & $83 \pm 6$ \\
M-NLC-RFB & $90 \pm 4$ \\
\hline
\end{tabular}

Notes: All values represent the mean \pm standard deviation $(n=3)$. No statistically significant differences were observed between conjugated and unconjugated formulations $(P>0.05)$.

Abbreviations: NLC, nanostructured lipid carrier; M, mannose; RFB, rifabutin. high $\%$ of drug encapsulation is expected since NLC are one of the main systems for incorporation of lipophilic drugs, such as RFB. ${ }^{16,20}$

\section{Morphology evaluation}

Cryo-SEM imaging revealed spherical particles, which were uniform in shape and had smooth surfaces for all the formulations (Figure 1). The mean diameter was between 100 and $300 \mathrm{~nm}$, and there was no visible aggregation of the particles. These observations validate the results obtained with DLS. As can be seen in Figure 1, both NLC (Figure 1A) and M-NLC (Figure 1B) formulations exhibit similar morphologies, indicating that the method of mannosylation does not influence the particle shape. Furthermore, from Figure 1C, it can be observed that NLC loaded with RFB also exhibit spherical shapes, indicating that the method of drug encapsulation does not result in morphological changes.

\section{Functionalization of NLC with mannose FT-IR}

Second-derivative spectra were obtained (as previously reported) to enhance the resolution of the absorption bands. ${ }^{21}$ The functionalization of the NLC with mannose was executed by the opening of the mannose ring and subsequent reaction of its aldehyde group with free amine functional groups present over the surface of uncoated NLC. ${ }^{12}$ This led to the formation of Schiff's base $(-\mathrm{N}=\mathrm{CH}-)$, which was detected by FT-IR spectroscopy (Figure 2). Figure 2 shows the peak formed at approximately $1,647.78 \mathrm{~cm}^{-1}$, proving the formation of Schiff's base $(-\mathrm{N}=\mathrm{CH}-)$ in M-NLC formulations. Similar results were also obtained in the studies conducted by Jain et $\mathrm{al}^{12}$ and Nimje et al. ${ }^{22}$

\section{In vitro drug release kinetics}

The in vitro release of RFB from NLC and M-NLC was measured in simulated physiological dissolution mediums to predict the in vivo efficacy of the formulations. The results obtained were analyzed using mathematical models for drug release kinetics, which are detailed in the Supplementary materials.

The drug release from NLC and M-NLC occurs by a diffusion-controlled process described by the Higuchi model since the plots of the amount of drug released versus time was found to be linear, using this model with a correlation coefficient between 0.998 and 0.944 , for all the $\mathrm{pH}$ studied and both formulations (Tables S1 and S2).

In addition, independent of the $\mathrm{pH}$, both NLC-RFB and M-NLC-RFB showed a biphasic drug-release pattern with an initial burst release followed by a sustained drug release 

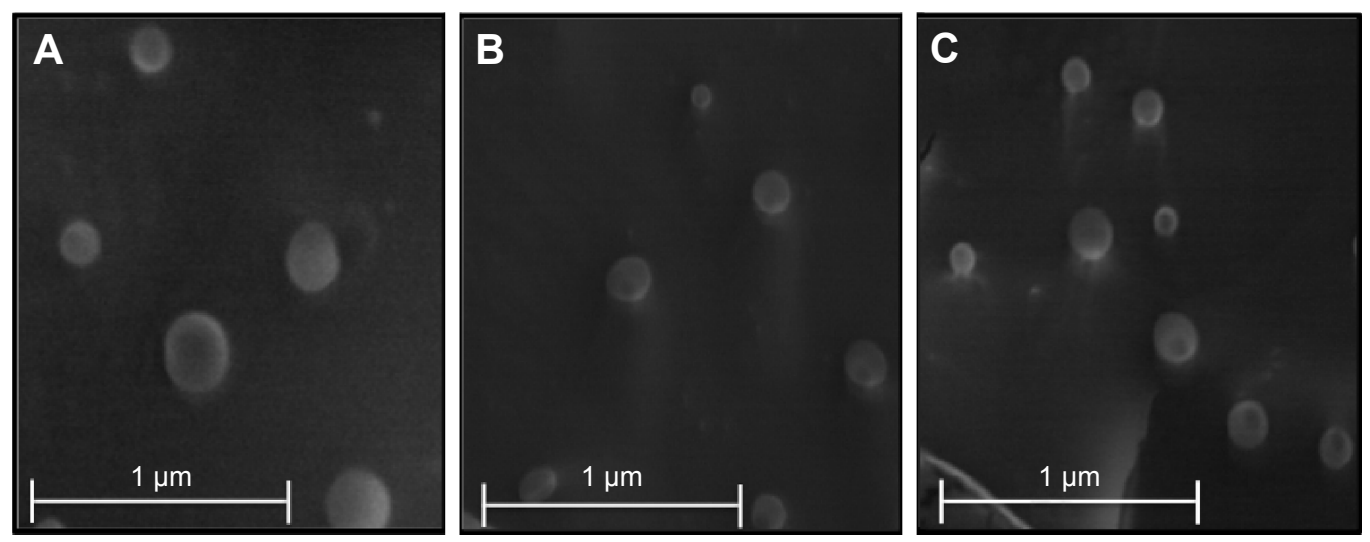

Figure I Cryo-SEM images of (A) NLC, (B) M-NLC, and (C) M-NLC-RFB at 20,000× magnification.

Abbreviations: NLC, nanostructured lipid carrier; M, mannose; RFB, rifabutin; SEM, scanning electron microscopy.

(Figure 3A and B). Moreover, a faster drug release profile was observed in the case of more acidic $\mathrm{pH}$ environments than at more neutral $\mathrm{pH}$ values for both formulations. Thus, the drug release of RFB from NLC and M-NLC are dependent on the $\mathrm{pH}$ of the environment. On the other hand, a slightly faster release of RFB was observed in the case of NLC compared to M-NLC due to the protective coat of mannose on the NLC surface. For instance, at $\mathrm{pH}=5.0$, a release of $50 \%$ of drug was achieved after 3 and 5 hours for NLC and M-NLC, respectively. Nevertheless, after 25 hours, an impressive $100 \%$ of drug release was achieved for both formulations at $\mathrm{pH}=5.0$, with only approximately $20 \%$ of the drug being released at $\mathrm{pH}=7.4$ for the same time frame.

Therefore, both formulations are suitable for RFB incorporation, and a much higher drug release occurs at acidic $\mathrm{pH}$ values, where the etiological agent of TB resides inside the infected cells, with the drug release being much lower at

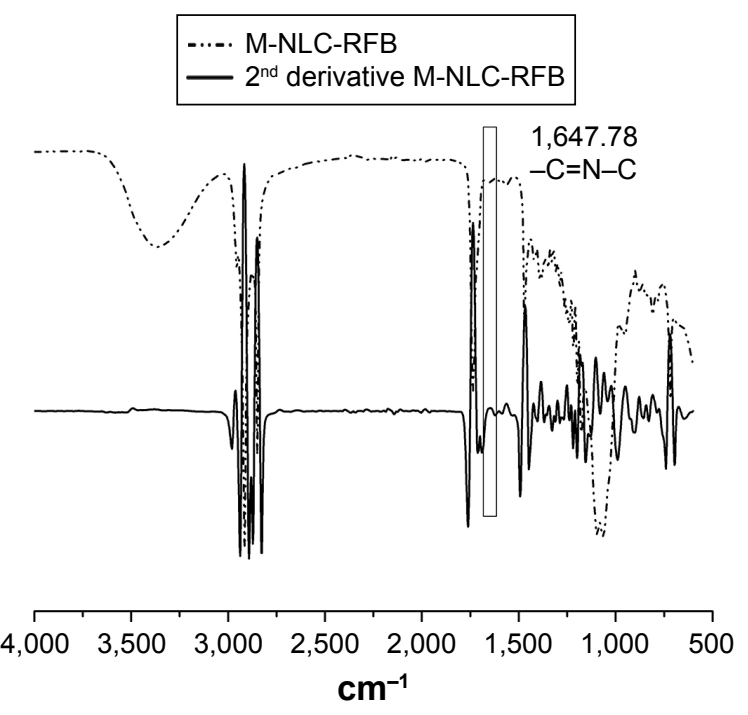

Figure 2 Second-derivative FT-IR spectra of M-NLC-RFB formulation.

Abbreviations: FT-IR, Fourier transform infrared; M, mannose; RFB, rifabutin; NLC, nanostructured lipid carrier. more neutral $\mathrm{pH}$ values, preventing the drug release in the noninfected cells.

\section{Effect of NLC on cell viability}

The effect of NLC, M-NLC, and M-NLC-RFB formulations on cell viability after 24 hours of incubation was studied in vitro on Calu-3, A549, and RAW 264.7 cells, through MTT assay. These three different cell lines were chosen to assess the cell viability of each formulation on the upper (Calu-3) and lower (A549) airway, as well as in the AMs (RAW 264.7). The half-maximum inhibitory concentration $\left(\mathrm{IC}_{50}\right)$ of each formulation obtained for all cell lines is listed in Table 4. All formulations showed a concentration-dependent effect, with toxicity increasing proportional to the concentration (Table 4). In fact, $\mathrm{IC}_{50}$ obtained for all formulations except for M-NLC and cell lines was above $100 \mu \mathrm{g} \mathrm{mL}^{-1}\left(\log _{10}\left(\mathrm{IC}_{50}\right)>2\right)$, but below $1,000 \mu \mathrm{g} \mathrm{mL}^{-1}\left(\log _{10}\left(\mathrm{IC}_{50}\right)<3\right)$. In the case of M-NLC and for all cell lines, an $\mathrm{IC}_{50}$ below $100 \mu \mathrm{g} \mathrm{mL}-1 \log _{10}\left(\mathrm{IC}_{50}\right)$ $<2$ ) was obtained. This may be explained by the surface charge of the nanoparticles. In fact, it is well known that the nanoparticles with positive charge are more toxic, ${ }^{23}$ the positive charge of the unloaded M-NLC was more pronounced than the positive charge of the drug-loaded M-NLC. The $\mathrm{IC}_{50}$ obtained for M-NLC-RFB was 238.9, 185.7, and 108.7 $\mu \mathrm{g} \mathrm{mL}^{-1}$ for Calu-3, A549, and RAW, respectively. Taking into consideration the $\mathrm{IC}_{50}$ obtained for M-NLC-RFB for the studied cell lines and regarding the drug content of $1 \%(\mathrm{w} / \mathrm{w})$, it is possible, with these formulations, to achieve maximum drug concentrations of $1.08 \mu \mathrm{g} \mathrm{mL}^{-1}$ at the site of delivery, which is reported to be a bactericidal concentration. ${ }^{24}$

\section{Long-term stability}

The physical stability of the NLC-RFB and M-NLC-RFB powder lyophilized formulations was verified periodically by analyzing the variation of the hydrodynamic diameter, PDI, 
A

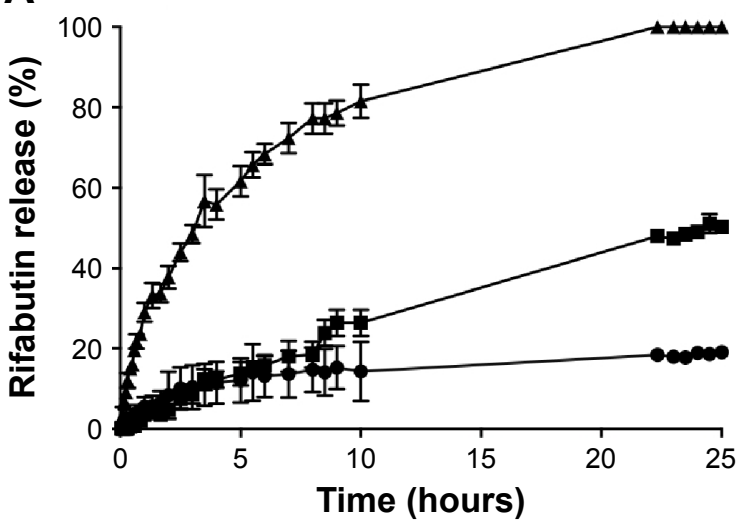

B

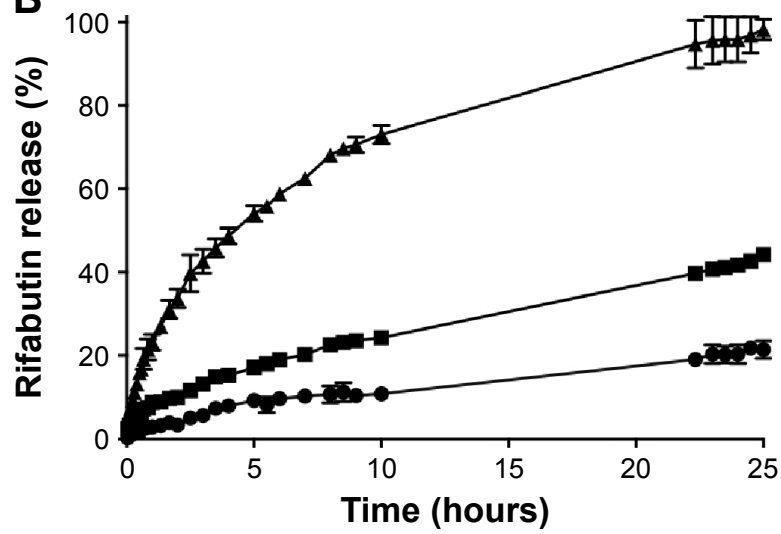

$\star \mathrm{pH}=5.0 \rightarrow \mathrm{pH}=6.2 \rightarrow \mathrm{pH}=7.4$

Figure 3 In vitro RFB release profiles from $\mathrm{NLC}(\mathbf{A})$ and $\mathrm{M}-\mathrm{NLC}(\mathbf{B})$ at three different $\mathrm{pH}$ values, $\mathrm{pH}=5.0,6.2$, and 7.4 , at body temperature $\left(37^{\circ} \mathrm{C}\right)$. Abbreviations: RFB, rifabutin; NLC, nanostructured lipid carrier.

and $\zeta$-potential during storage conditions for 6 months at room temperature (Table 5). The main characteristic changed after the lyophilization process was the alteration of the $\zeta$-potential of the M-NLC-RFB, which shifted from positive to negative values (Table 5). This is due to the presence of the cryoprotectant Aerosil in the final formulation, which itself has a negative charge, thus, increasing the absolute values of the $\zeta$-potential of the nanoparticles. ${ }^{17}$ Moreover, as shown in Table 5, no tendency to $\zeta$-potential variations was observed for the nonmannosylated and mannosylated loaded NLC, reinforcing the long-term stability of the developed nanoparticles. Additionally, the hydrodynamic diameter and PDI of the NLC-RFB and M-NLC-RFB formulations only suffers a small variation over time. Contrastingly, a more pronounced effect in the hydrodynamic diameter and PDI is observed for NLC-RFB formulations. Therefore, the developed NLC are suitable for RFB incorporation and for preventing the release of the drug during storage at room temperature.

\section{Conclusion}

This study focused on the development of NLC for the incorporation of RFB to selectively target the infected AMs

Table 4 Cell viability in RAW, A549, and Calu- 3 cells as a function of NLC, M-NLC, and M-NLC-RFB in concentrations of the lyophilized formulations ( $1,10,100$, and $1,000 \mathrm{mg} / \mathrm{mL}$ ) expressed in $\log _{10}$

\begin{tabular}{llll}
\hline Formulations & NLC & M-NLC & M-NLC-RFB \\
\hline Calu-3 & $2.706 \pm 0.178$ & $1.482 \pm 0.772$ & $2.378 \pm 0.253$ \\
A549 & $2.259 \pm 0.095$ & $1.272 \pm 0.452$ & $2.269 \pm 0.592$ \\
RAW & $2.120 \pm 0.267$ & $1.276 \pm 0.654$ & $2.036 \pm 0.592$ \\
\hline
\end{tabular}

Notes: All values represent the mean \pm standard deviation $(n=3)$. No statistically significant differences were observed between unconjugated and conjugated loaded and unloaded formulations $(P>0.05)$

Abbreviations: NLC, nanostructured lipid carrier; M, mannose; RFB, rifabutin. and thus improve RFB's therapeutic index. Mannose surface modification of NLC was made to take advantage of the presence of sugar receptors in the target AMs, which will improve cellular uptake by an active targeting strategy. Drug release from the developed formulations are $\mathrm{pH}$ sensitive, which can be of particular interest for releasing the drug mainly under the acidic conditions seen in phagosomes ( $\mathrm{pH} \sim 6.2$ ) and phagolysosomes $(\mathrm{pH} \sim 5.0$ ), where the etiologic agent of TB is located. The difference in the $\zeta$-potential between the uncoated and the mannosylated NLC constituted evidence that the mannosylation process was successful. The mannosylation process was confirmed by FT-IR spectrum analysis, allowing detection of the presence of the Schiff's base in the coated formulations.

The diameter of the produced particles was determined by DLS and found to be around $200 \mathrm{~nm}$. This diameter is considered to be appropriate for lung deposition, validating the proposed route of administration, namely the pulmonary route. ${ }^{17}$ Due to their diameter, it is expected that NLC could

Table 5 Characterization of the hydrodynamic diameter, polydispersity index, and zeta-potential after 0 , I, and 6 months of the synthesis of NLC-RFB and M-NLC-RFB lyophilized formulations

\begin{tabular}{lllll}
\hline Formulations Time & $\begin{array}{l}\text { Mean } \\
\text { (months) } \\
\text { hydrodynamic } \\
\text { diameter }(\mathbf{n m})\end{array}$ & $\begin{array}{l}\text { Polydispersity } \\
\text { index (PDI) }\end{array}$ & $\begin{array}{l}\text { Zeta- } \\
\text { potential } \\
(\mathbf{m V})\end{array}$ \\
\hline NLC-RFB & 0 & $200 \pm 2$ & $0.205 \pm 0.010$ & $-29 \pm 2$ \\
& 1 & $191 \pm 1$ & $0.249 \pm 0.006$ & $-28 \pm 1$ \\
& 6 & $248 \pm 2$ & $0.212 \pm 0.012$ & $-28 \pm 1$ \\
M-NLC-RFB & 0 & $198 \pm 2$ & $0.211 \pm 0.010$ & $-27 \pm 1$ \\
& 1 & $192 \pm 3$ & $0.266 \pm 0.006$ & $-24 \pm 1$ \\
& 6 & $20 I \pm 2$ & $0.197 \pm 0.008$ & $-32 \pm 2$ \\
\hline
\end{tabular}

Abbreviations: RFB, rifabutin; NLC, nanostructured lipid carrier; M, mannose. 
reach the pulmonary alveoli by passive targeting. In addition, the $\zeta$-potential was considerably high for all formulations, which suggested a fairly good shelf life stability. Morphology studies showed spherical and uniform nanoparticles with smooth surfaces. The diameter of the NLC studied by cryoSEM were also consistent with DLS analysis. The percentage of encapsulation of RFB was found to be high, with an EE above $80 \%$ for both uncoated and mannose-coated NLC. Finally, the effect of the NLC formulations on cell viability was performed using the MTT assay with different cell lines (Calu-3, A549, and RAW 264.7). The overall results confirmed that it was possible to reach concentrations above 100 and below $1,000 \mu \mathrm{g} \mathrm{mL}^{-1}$ before $\mathrm{IC}_{50}$ was reached, which is sufficient to attain therapeutic concentrations of this drug. ${ }^{24}$ Moreover, the release rate of RFB from the nanocarriers was faster at acidic $\mathrm{pH}$ values than at neutral $\mathrm{pH}$. Since the macrophages are particularly efficient in internalizing particles and delivering the particles to acidified phagosomes and phagolysosomes, these results strongly suggest that the release of RFB will be higher intracellularly at these acidified structures where the MTb resides. Additionally, the stability study demonstrated physical stability for a period of at least 6 months.

The results obtained in this study pose a strong argument that NLC constitute a promising strategy for the pulmonary delivery of RFB selectively to the AMs. ${ }^{11}$ Further work is required to validate the clinical potential of the developed formulations since these results are very promising and can open further avenues to effectively fight TB.

\section{Acknowledgments}

Alexandre Vieira thanks the CNPq, Ministry of Education of Brazil for the fellowship 246514/2012-4. Marina Pinheiro is thankful to Fundação para a Ciência e Tecnologia (FCT) for the Post-Doctorate Grant (SFRH/BPD/99124/2013). Additionally, this work was possible with financial support from FCT/MEC through National Funds and co-financed by FEDER, under the partnership agreement PT2020 - UID/ MULTI/04378/2013 -POCI/01/0145/FERDER/007728.

\section{Disclosure}

The authors report no conflicts of interest in this work.

\section{References}

1. Abubakar I, Zignol M, Falzon D, et al. Drug-resistant tuberculosis: time for visionary political leadership. Lancet Infect Dis. 2013;13(6): 529-539.

2. World Health Organization. Global Tuberculosis Report 2014. Geneva, Switzerland: World Health Organization; 2014:1-171.
3. Pinheiro M, Lúcio M, Lima J, Reis S. Liposomes as drug delivery systems for the treatment of TB. Nanomedicine (Lond). 2011;6(8):1413-1428.

4. Muller RH, Mader K, Gohla S. Solid lipid nanoparticles (SLN) for controlled drug delivery - a review of the state of the art. Eur J Pharm Biopharm. 2000;50(1):161-177.

5. Muller RH, Radtke M, Wissing SA. Solid lipid nanoparticles (SLN) and nanostructured lipid carriers (NLC) in cosmetic and dermatological preparations. Adv Drug Deliv Rev. 2002;54(Suppl 1):S131-S155.

6. Muller RH, Keck CM. Challenges and solutions for the delivery of biotech drugs - a review of drug nanocrystal technology and lipid nanoparticles. J Biotechnol. 2004;113(1-3):151-170.

7. Mehnert W, Mader K. Solid lipid nanoparticles: production, characterization and applications. Adv Drug Deliv Rev. 2001;47(2-3):165-196.

8. Song X, Lin Q, Guo L, et al. Rifampicin loaded mannosylated cationic nanostructured lipid carriers for alveolar macrophage-specific delivery. Pharm Res. 2015;32(5):1741-1751.

9. Aristoff PA, Garcia GA, Kirchhoff PD, Showalter HD. Rifamycins obstacles and opportunities. Tuberculosis (Edinb). 2010;90(2):94-118.

10. Irache JM, Salman HH, Gamazo C, Espuelas S. Mannose-targeted systems for the delivery of therapeutics. Expert Opin Drug Deliv. 2008; 5(6):703-724.

11. Ribeiro R. Development and Characterization of Nanocarrier Systems for the Delivery of Antitubercular Drugs. Porto, Pourtgal: Universidade do Porto; 2014.

12. Jain A, Agarwal A, Majumder S, et al. Mannosylated solid lipid nanoparticles as vectors for site-specific delivery of an anti-cancer drug. $J$ Control Release. 2010;148(3):359-367.

13. Urgently needed: new antibiotics. Lancet. 2009;374(9705):1868.

14. Varshosaz J, Eskandari S, Tabbakhian M. Freeze-drying of nanostructure lipid carriers by different carbohydrate polymers used as cryoprotectants. Carbohydr Polym. 2012;88(4):1157-1163.

15. Danhier F, Lecouturier N, Vroman B, et al. Paclitaxel-loaded PEGylated PLGA-based nanoparticles: in vitro and in vivo evaluation. $J$ Control Release. 2009;133(1):11-17.

16. Pinheiro M, Pisco S, Silva AS, Nunes C, Reis S. Evaluation of the effect of rifampicin on the biophysical properties of the membranes: significance for therapeutic and side effects. Int J Pharm. 2014;466(1-2): 190-197.

17. Chow AH, Tong HH, Chattopadhyay P, Shekunov BY. Particle engineering for pulmonary drug delivery. Pharm Res. 2007;24(3):411-437.

18. Cho M, Cho WS, Choi M, et al. The impact of size on tissue distribution and elimination by single intravenous injection of silica nanoparticles. Toxicol Lett. 2009;189(3):177-183.

19. Müller H, Jacobs C, Kayser O. Nanosuspensions as particulate drug formulations in therapy: rationale for development and what we can expect for the future. Adv Drug Deliv Rev. 2001;23(1):3-19.

20. Das S, Chaudhury A. Recent advances in lipid nanoparticle formulations with solid matrix for oral drug delivery. AAPS PharmSciTech. 2011;12(1):62-76.

21. Kong J, Yu S. Fourier transform infrared spectroscopic analysis of protein secondary structures. Acta Biochim Biophys Sin (Shanghai). 2007; 39(8):549-559.

22. Nimje N, Agarwal A, Saraogi GK, et al. Mannosylated nanoparticulate carriers of rifabutin for alveolar targeting. J Drug Target. 2009;17(10): 777-787.

23. Kedmi R, Ben-Arie N, Peer D. The systemic toxicity of positively charged lipid nanoparticles and the role of Toll-like receptor 4 in immune activation. Biomaterials. 2010;31(26):6867-6875.

24. Kunin CM. Antimicrobial activity of rifabutin, clinical infectious diseases: an official publication of the Infectious Diseases Society of America. Clin Infect Dis. 1996;22(Suppl 1):S3-S13; discussion S13-S14. 


\section{Supplementary materials}

Table SI Value of $R^{2}$ obtained from the release of RFB from the NLC-RFB lyophilized formulation for different models of mechanism of drug release

\begin{tabular}{llllll}
\hline pH & $\begin{array}{l}\text { Zero } \\
\text { order }\end{array}$ & $\begin{array}{l}\text { First } \\
\text { order }\end{array}$ & $\begin{array}{l}\text { Hixson- } \\
\text { Crowell }\end{array}$ & Higuchi & $\begin{array}{l}\text { Korsmeyer- } \\
\text { Peppas }\end{array}$ \\
\hline 5.0 & 0.919 & 0.655 & 0.660 & 0.993 & 0.743 \\
6.2 & 0.968 & 0.839 & 0.899 & 0.981 & 0.969 \\
7.4 & 0.821 & 0.506 & 0.660 & 0.944 & 0.801 \\
\hline
\end{tabular}

Abbreviations: RFB, rifabutin; NLC, nanostructured lipid carrier.
Table S2 Value of $R^{2}$ obtained from the release of RFB from the M-NLC-RFB lyophilized formulation for different models of mechanism of drug release

\begin{tabular}{llllll}
\hline pH & $\begin{array}{l}\text { Zero } \\
\text { order }\end{array}$ & $\begin{array}{l}\text { First } \\
\text { order }\end{array}$ & $\begin{array}{l}\text { Hixson- } \\
\text { Crowell }\end{array}$ & Higuchi & $\begin{array}{l}\text { Korsmeyer- } \\
\text { Peppas }\end{array}$ \\
\hline 5.0 & 0.934 & 0.780 & 0.841 & 0.998 & 0.935 \\
6.2 & 0.978 & 0.831 & 0.894 & 0.993 & 0.967 \\
7.4 & 0.919 & 0.651 & 0.789 & 0.944 & 0.931 \\
\hline
\end{tabular}

Abbreviations: RFB, rifabutin; M, mannose; NLC, nanostructured lipid carrier.

\section{Publish your work in this journal}

Drug Design, Development and Therapy is an international, peerreviewed open-access journal that spans the spectrum of drug design and development through to clinical applications. Clinical outcomes, patient safety, and programs for the development and effective, safe, and sustained use of medicines are a feature of the journal, which has also been accepted for indexing on PubMed Central. The manuscript management system is completely online and includes a very quick and fair peer-review system, which is all easy to use. Visit http://www.dovepress.com/testimonials.php to read real quotes from published authors.

Submit your manuscript here: http://www.dovepress.com/drug-design-development-and-therapy-journal 